\title{
INDICADORES DE ESTRESSE, ANSIEDADE E DEPRESSÃO EM PAIS DE CRIANÇAS DIAGNOSTICADAS COM TEA
}

\section{INDICATORS OF STRESS, ANXIETY AND DEPRESSION IN PARENTS OF CHILDREN DIAGNOSED WITH ASD}

\author{
Luena Lima Alves ${ }^{1}$, Ana Flávia Lima Teles Da Hora ${ }^{2}$
}

\begin{abstract}
RESUMO: O Transtorno do Espectro Autista (TEA) é uma síndrome do neurodesenvolvimento, caracterizada por dificuldades nas áreas da socialização, comunicação e comportamento que, segundo a Organização Mundial da Saúde (OMS, 2017), 1 a cada 160 pessoas a possuem. O diagnóstico de autismo pode causar grandes impactos na família e, principalmente, nos pais, uma vez que o desconhecido traz consigo vários sentimentos e questionamentos. Ademais, pode desencadear estresse e transtornos de ansiedade e depressão. Metodologicamente, é uma pesquisa exploratória, quantitativa, transversal, caracterizada como um estudo preliminar que tem por objetivo identificar nos pais de crianças autistas o grau dos indicadores de estresse, ansiedade e depressão, manifestados por esses genitores. Utilizou-se como instrumento a Escala de Ansiedade, Depressão e Estresse (EADS-21) em uma amostra pequena, composta por 7 pais atendidos no Laboratório de Avaliação, Pesquisa e Intervenção em Transtorno do Espectro Autista da Universidade CEUMA. Os resultados foram analisados de forma quantitativa pelo programa estatístico SPSS (Statistical Package for Social Sciense), versão 22.0, os quais revelam que dos 7 participantes, 1 mãe apresentou sintomas de estresse leve e ansiedade moderada e 1 pai apresentou ansiedade moderada. $\mathrm{O}$ estudo contribuiu para a obtenção de conhecimento acerca dos agravantes que o diagnóstico de autismo pode trazer para os genitores, bem como as consequências para a sua qualidade de vida.
\end{abstract}

PALAVRAS-CHAVE: Autismo; Família; Estresse; Ansiedade; Depressão.

ABSTRACT: Autistic Spectrum Disorder (ASD) is a neurodevelopmental syndrome characterized by difficulties in the areas of socialization, communication and behavior, according to the World Health Organization (WHO, 2017), 1 in 160 people have it. The diagnosis of autism can have great impacts on the family and especially on the parents, since the unknown brings with it several feelings and questions. In addition, it can trigger stress and anxiety disorders and depression. Methodologically, it is an exploratory, quantitative, transversal research, characterized as a preliminary study that aims to identify in the parents of autistic children the degree of indicators of anxiety, depression and stress manifested by these parents. The EADS-21 was used as an instrument in a small sample composed of 7 parents attending the Laboratory of Evaluation, Research and Intervention in Autism Spectrum Disorder at Ceuma University. The results were analyzed quantitatively by the statistical program SPSS

\footnotetext{
${ }^{1}$ Graduanda do curso de Psicologia, cursando o $10^{\circ}$ período na Universidade Ceuma. E-mail: luena.alves@hotmail.com

${ }^{2}$ Psicóloga. Doutora e Mestre em Teoria e Pesquisa do Comportamento pela Universidade Federal do Pará (UFPA). Professora e pesquisadora na Universidade Ceuma e no Núcleo de Tecnologia para Educação (UEMANET) da Universidade Estadual do Maranhão. E-mail: anaflaviadahora@ @otmail.com
} 
(Statistical Package for Social Sciense), version 22.0, which reveal that of the 7 participants, 1 mother had symptoms of mild stress and moderate anxiety and 1 father presented moderate anxiety. Due to the small size of the clinical sample, it is important that such results be evaluated with caution, and further research with larger clinical specimens is indispensable. The study contributed to gain knowledge about the aggravating factors that the diagnosis of autism can bring to the parents, as well as the consequences for their quality of life.

KEYWORDS: Autism; Family; Stress; Anxiety; Depression.

\section{INTRODUÇÃO}

No cenário atual, sabe-se que o autismo, síndrome que compromete o desenvolvimento neurológico, a interação social, o comportamento e a comunicação, pode atingir uma a cada cinquenta crianças, tendo maior prevalência no sexo masculino, na proporção de três homens para uma mulher, além de apresentar o nível de comprometimento, os quais podem ser grau leve, moderado ou severo (CÉSAR, 2015).

O diagnóstico do TEA, assim como o de qualquer outra síndrome, atinge toda a família da criança, principalmente os pais, que aguardam ansiosamente pela chegada de um filho absolutamente saudável, sem qualquer deficiência. Tal diagnóstico causa nos pais sentimentos de fracasso, visto que a criança ideal não nasceu conforme o desejado (JERUSALINSKY, 2007). Assim, podem surgir nos genitores agravantes de estresse, ansiedade ou depressão, que consequentemente prejudicarão a qualidade de vida destes e até do próprio filho, uma vez que a dinâmica familiar foi alterada.

Sardá Jr., Legal e Jablonski Jr., (2004) afirmam que o estresse caracteriza-se como uma resposta saudável não específica do organismo frente alguma situação que venha ameaçar o equilíbrio do corpo, ou seja, a homeostase. Assim, o estresse pode acometer todo e qualquer sujeito. Contudo, é necessário analisar a magnitude, a continuidade e a frequência dos aspetos estressantes, uma vez que a reação deixa de ser saudável e passa a ser patológica, quando tal estresse afeta o bem-estar do indivíduo e este não consegue mais voltar ao seu estado de equilíbrio.

Logo, fatores como a limitação na comunicação das crianças autistas, os seus problemas de comportamento, bem como a incapacidade de se relacionarem socialmente, a hiperatividade, o isolamento, a irritação e a gravidade dos seus sintomas contribuem não só para o estresse materno dessas genitoras, como paterno também, visto que há um aumento considerável no nível de estresse dessas famílias. Ademais, 
quanto aos transtornos, os pais estão propensos a desenvolver ansiedade e/ou depressão (AMARAL; CAVALCANTE; SILVA, 2013).

Ao se tratar de ansiedade, sabe-se que esta é uma emoção que está presente na vida de todos os sujeitos, caracterizando-se como um sentimento incômodo de medo, de estranheza, os quais, geralmente, advêm de uma situação nova. De acordo com a OMS (2017), 9,3\% dos brasileiros possuem algum transtorno de ansiedade. Assim, é importante que se faça uma distinção entre a ansiedade considerada normal e a patológica. Desse modo, é necessário investigar a duração da resposta ansiosa, ou seja, se é breve em relação à ocasião em questão ou desproporcional, capaz de afetar a produção diária dos indivíduos, bem como a sua qualidade de vida ou estabilidade emocional (CASTILLO et al., 2000).

Em continuidade, outra patologia que traz prejuízos aos sujeitos é a depressão. Conforme o DSM-5 (2014), os indivíduos acometidos pela depressão apresentam normalmente sintomas como humor deprimido em grande parte do dia, ou seja, sentemse tristes, desesperançados, desprazer, distúrbios de apetite (emagrecer ou engordar com facilidade) e sono (insônia ou hipersônia), sentimentos de culpa, baixa autoestima e pensamentos recorrentes de morte. Ademais, a depressão compromete a maneira como o indivíduo se compreende, ou seja, modifica a percepção que possui de si mesmo, além de afetar o seu modo de se relacionar com o mundo, nesse caso, mães e pais.

Desse modo, o estado emocional desses pais é um fator importante a ser levado em consideração, visto que as crianças em fase de crescimento são totalmente dependentes de seus genitores quanto à estimulação intelectual e ao exercício do convívio social, principalmente as crianças autistas. O TEA provoca mudanças em toda a família e altera toda a sua dinâmica. É um processo longo. No entanto, é possível se adaptar às mudanças sem deixar os seus desejos e necessidades em segundo plano e, a partir disso, ter uma boa qualidade de vida (QV), ultrapassando as situações de estresse, ansiedade e depressão.

\section{MATERIAIS E MÉTODOS}

O presente estudo caracteriza-se como uma pesquisa de natureza descritiva que tem como finalidade oportunizar maior conhecimento acerca do problema, com efeito de elaborar proposições ou torná-lo mais compreensível (GIL, 2007). Tal pesquisa se dispõe a investigar os indicadores de ansiedade, depressão e estresse em pais de crianças 
diagnosticadas com TEA, visto que tais fatores têm se tornado cada vez mais presentes nos dias atuais e têm atingido, gradativamente, os pais de filhos autistas.

A pesquisa foi realizada no Laboratório de Avaliação, Pesquisa e Intervenção em Transtorno do Espectro Autista (LAPITEA), localizado na Universidade CEUMA, São Luís - MA. Foi uma amostra clínica pequena, composta por 7 pais de crianças diagnosticadas clinicamente com TEA, com idades variantes de 6 a 12 anos, sendo 4 sujeitos do sexo masculino e 3 do sexo feminino. $\mathrm{O}$ instrumento utilizado para avaliar o nível de ansiedade, depressão e estresse foi a Escala de Ansiedade, Depressão e Estresse (EADS-21), que consiste em uma ferramenta constituída por três subescalas, as quais possuem cada uma sete itens de múltipla escolha, totalizando vinte e um itens que visam avaliar as manifestações clínicas desses três estados emocionais.

Os dados foram coletados em salas de atendimentos da clínica escola com a autorização da orientadora. Previamente à aplicação do instrumento, explicou-se o objetivo da pesquisa aos participantes, bem como a garantia do anonimato e o sigilo profissional, prática pertencente ao trabalho do psicólogo. A análise de dados foi realizada pelo programa estatístico SPSS-20 (Statistical Package for the Social Sciences), versão 22.0, de forma descritiva, com respostas obtidas pela contagem de pontos em cada item do instrumento. Os resultados serão apresentados a seguir por meio de tabela e gráfico com o propósito de efetivar os objetivos dessa pesquisa. A presente pesquisa integra um projeto guarda-chuva do LAPITEA, com o registro de aprovação do Comitê de Ética em Pesquisa (CEP) da Universidade CEUMA de CAAE: 70185617.1.0000.5084.

\section{RESULTADOS}

Na Tabela 1, apresentam-se a média e os desvios padrão dos sintomas de cada um dos sete itens que compõem as subescalas da EADS-21. E o gráfico 1 seguinte apresenta a frequência dos sintomas na amostra: 
Tabela 1. Frequência dos sintomas da escala

\begin{tabular}{|l|c|c|c|c|c|}
\hline \multicolumn{7}{|c|}{ Escala de Estresse } \\
\hline \multicolumn{1}{|c|}{ Sintomas } & N & Mínimo & Máximo & $\begin{array}{c}\text { Média } \\
\text { (M) }\end{array}$ & $\begin{array}{c}\text { Desvio } \\
\text { Padrão (DP) }\end{array}$ \\
\hline 2. Minha boca ficou seca & 7 & 0 & 3 & 1,29 & 1,254 \\
\hline $\begin{array}{l}\text { 4. Em alguns momentos, tive dificuldade } \\
\text { de respirar (chiado e falta de ar sem } \\
\text { esforço físico) }\end{array}$ & 7 & 0 & 2 & 0,57 & 0,787 \\
\hline $\begin{array}{l}\text { 7. Senti tremedeira (por exemplo, nas } \\
\text { mãos) }\end{array}$ & 7 & 0 & 2 & 0,86 & 0,900 \\
\hline $\begin{array}{l}\text { 9. Me preocupei com situações em que } \\
\text { poderia entrar em pânico e parecer } \\
\text { ridículo (a) }\end{array}$ & 7 & 0 & 2 & 0,43 & 0,787 \\
\hline 15. Eu senti que iria entrar em pânico & 7 & 0 & 1 & 0,29 & 0,488 \\
\hline $\begin{array}{l}\text { 19. Eu percebi uma mudança nos meus } \\
\text { batimentos cardíacos, embora não } \\
\text { estivesse praticando exercício rigoroso } \\
\text { (ex. batimento cardíaco acelerado ou }\end{array}$ & 7 & & & & 0,951 \\
irregular) & & 0 & 2 & 0,71 & 0,787 \\
\hline 20. Eu senti medo sem motivo
\end{tabular}

\section{Escala de Ansiedade}

\begin{tabular}{|l|r|r|r|r|r|}
\hline 1. Tive dificuldade de me acalmar & 7 & 0 & 3 & 1,00 & 1,155 \\
\hline $\begin{array}{l}\text { 6. Exagerei intencionalmente ao reagir às } \\
\text { situações }\end{array}$ & 7 & 0 & 2 & 0,86 & 0,690 \\
\hline 8. Senti que estava sempre nervoso (a) & 7 & 0 & 2 & 0,57 & 0,787 \\
\hline 11. Me senti inquieto (a) & 7 & 0 & 1 & 0,43 & 0,535 \\
\hline 12. Tive dificuldade de relaxar & 7 & 0 & 3 & 1,29 & 0,951 \\
\hline $\begin{array}{l}\text { 14. Eu não conseguia tolerar as coisas que } \\
\text { me impediam de continuar a fazer o que } \\
\text { estava realizando }\end{array}$ & 7 & 0 & 2 & 0,86 & 0,690 \\
\hline $\begin{array}{l}\text { 18. Eu senti que estava sendo muito } \\
\text { sensível/emotivo }\end{array}$ & 7 & 0 & 2 & 0,71 & 0,756 \\
\hline
\end{tabular}

Escala de Depressão

\begin{tabular}{|l|c|r|r|r|r|}
\hline 3. Não tive sentimento positivo & 7 & 0 & 3 & 1,00 & 1,000 \\
\hline $\begin{array}{l}\text { 5. Não consegui ter iniciativa para fazer as } \\
\text { coisas }\end{array}$ & 7 & 0 & 3 & 1,43 & 1,134 \\
\hline 10. Senti que não tinha vontade de nada & 7 & 0 & 2 & 0,71 & 0,756 \\
\hline 13. Me senti deprimido e sem motivação & 7 & 0 & 1 & 0,86 & 0,378 \\
\hline 16. Nada me deixou entusiasmado & 7 & 0 & 1 & 0,57 & 0,535 \\
\hline 17. Eu senti que era uma pessoa sem valor & 7 & 0 & 1 & 0,43 & 0,535 \\
\hline 21. Senti que a vida não tinha sentido & 7 & 0 & 1 & 0,14 & 0,378 \\
\hline
\end{tabular}

\section{Fonte: Autores}




\section{Gráfico 1. Frequência dos sintomas na amostra}

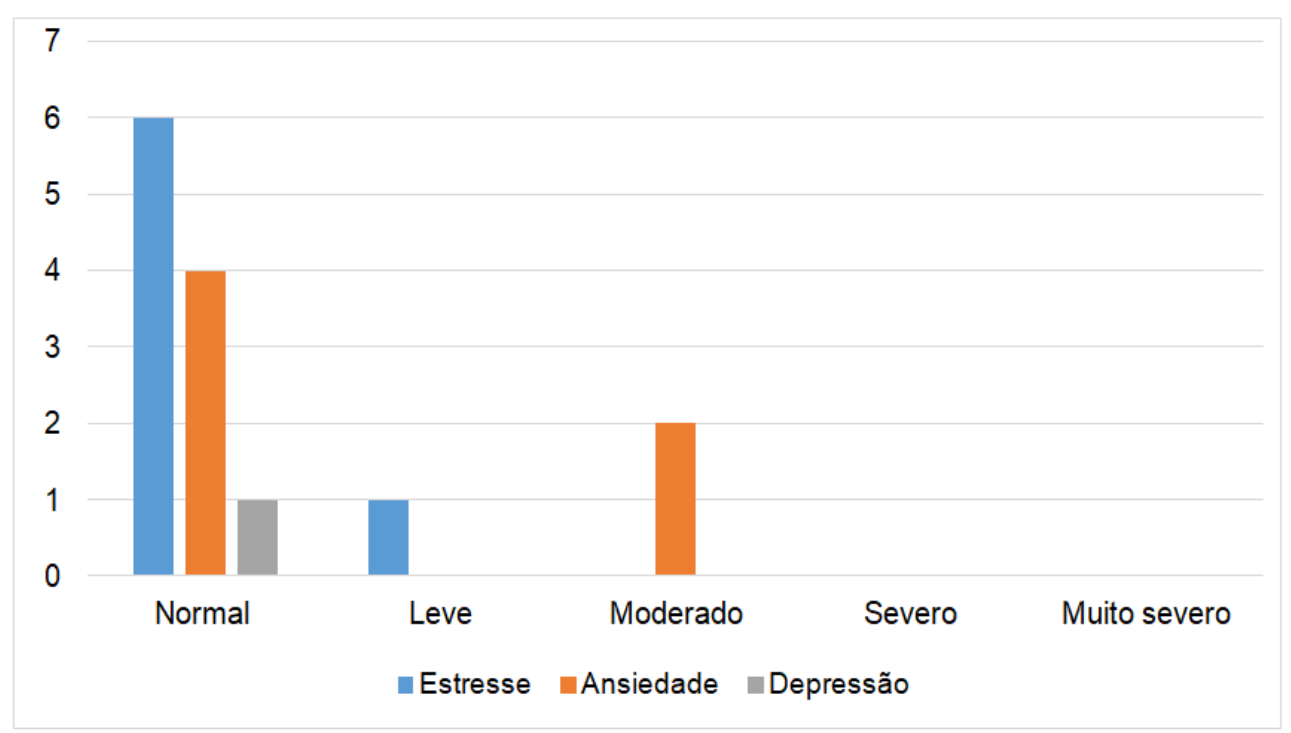

Fonte: Autores

Conforme exposto na Tabela 1, na escala de estresse, os valores de média variam entre 0,29 (item $15=$ Eu senti que ia entrar em pânico) e 1,29 (item $2=$ Minha boca ficou seca). Os sintomas que apresentaram as maiores médias: 0,71 (item $=19$. Eu percebi uma mudança nos meus batimentos cardíacos, embora não estivesse praticando exercício rigoroso, - ex: batimento cardíaco acelerado ou irregular-); 0,86 (item $=7$. Senti tremedeira - por exemplo, nas mãos -); e 1,29 (item 2 = Minha boca ficou seca), sintoma que apresentou maior média.

$\mathrm{Na}$ escala de ansiedade, houve uma variância de valores entre 0,43 (item $11=$ Me senti inquieto (a)) e 1,29 (item 12 = Tive dificuldade de relaxar). Nessa escala, os sintomas presentes na amostra estudada que apresentaram maiores médias foram: 0,71 (item 18 = Eu senti que estava sendo muito sensível/emotivo); 0,86 (itens 6 e 14 respectivamente $=$ Exagerei intencionalmente ao reagir às situações / Eu não conseguia tolerar as coisas que me impediam de continuar a fazer o que estava realizando); 1,00 (item 1 = Tive dificuldade de me acalmar) e 1,29 (item 12 = Tive dificuldade de relaxar).

Já na escala de depressão, os valores encontrados variam entre 0,14 (item $21=$ Senti que a vida não tinha sentido) e 1,43 (item 5 = Não consegui ter iniciativa para 
fazer as coisas). Os sintomas com maiores médias: 0,71 (item $10=$ Senti que não tinha vontade de nada); 0,86 (item $13=$ Me senti deprimido e sem motivação); 1,00 (item $3=$ Não tive sentimento positivo) e 1,43 (item 5 = Não consegui ter iniciativa para fazer as coisas).

No Quadro 1, observa-se que apenas uma mãe apresentou sintomas de estresse leve e ansiedade moderada e um pai manifestou sintomas de ansiedade moderada.

\section{DISCUSSÃO}

A partir dos resultados preliminares apresentados neste estudo, realizado com uma amostra clínica considerada pequena, pode-se inferir que, dos 7 pais testados, 5 deles $(71,4 \%)$ não apresentaram indicadores de estresse, ansiedade ou depressão, e que 2 pais $(28,5 \%)$ apresentaram sintomas de estresse leve e ansiedade moderada.

Kinrys e Wygant (2005) em suas pesquisas apontam que as mulheres apresentam maiores chances de desenvolverem algum transtorno de ansiedade ao longo da vida se comparadas aos homens. Tal situação pode ser explicada pela presença dos hormônios sexuais femininos e ciclos menstruais que provocam alterações hormonais e, consequentemente. emocionais. Assim, fazendo uma comparação dos resultados encontrados no presente estudo, nota-se que uma mãe e um pai apresentaram sintomas de ansiedade moderada, confirmando a pesquisa dos autores citados.

Em relação à amostra, as crianças com TEA estão sendo assistidas pela metodologia Análise do Comportamento Aplicada (ABA), que conforme Lear (2004) é um termo "guarda-chuva" o qual caracteriza uma abordagem científica que pode ser utilizada para tratar numerosas demandas diferenciadas e cobrir variados tipos distintos de intervenções, particularmente educação especial para crianças com o espectro. Ao passo que os pais estão participando de oficinas as quais os ensinam sobre como modelar determinados comportamentos dos filhos.

Logo, tal envolvimento familiar possivelmente influencia nos baixos níveis de sintomas manifestados pelos pais, em comparação ao que afirmam Fávero e Santos (2005), em seu estudo de revisão bibliográfica acerca do autismo infantil e o estresse familiar, que os aconselhamentos, instruções, sobretudo o apoio social por meio das instituições de atendimento as crianças, e também os trabalhos terapêuticos, aparecem como possibilidades de enfrentamento, capazes de reduzir os sintomas referidos, por esta razão os resultados obtidos são considerados positivos. 
Ainda em concordância, outro estudo de revisão realizado por Bosa (2006), a autora disserta sobre os aspectos benéficos acerca das intervenções que os pais podem aprender no intuito de realizá-las com os filhos, afirmando que não basta apenas dizer aos genitores o que fazer, mas sim mostrá-los como fazer, como é o caso das oficinas realizadas com os pais no LAPITEA. Tal oficina traz benefícios não só para os pais, mas principalmente para as crianças.

$\mathrm{Na}$ literatura, encontram-se diversos resultados, como Schmidt e Bosa (2007), que efetuaram um estudo com 30 mulheres com idades entre 30 a 56 anos, com filhos diagnosticados com TEA, com idades entre 12 e 30 anos. Tal estudo objetivou analisar a presença de estresse e da autoeficácia nessas mães de crianças autistas. Então, os resultados obtidos revelaram que das 30 mães, 21 delas apresentaram indicadores de estresse, e 13 estavam na fase de resistência. Ademais, o estudo mostrou que as mães são as principais responsáveis pelos cuidados dos filhos.

Devido às complicações na área da comunicação das crianças autistas, ainda segundo o estudo dos autores citados acima, as genitoras expuseram sentir dificuldades para se comunicarem com os filhos no que tange a compreensão dos estados afetivos e sentimentos destes. No entanto, as ajudas recebidas de profissionais e também da família são fatores contribuintes para a diminuição dos sintomas estressores nas mães (SANCHEZ; BAPTISTA, 2009).

Outro estudo realizado por Sprovieri e Assumpção Jr. (2001), no qual avaliaram 45 famílias, sendo 15 famílias com crianças com TEA, 15 famílias com filhos portadores de Síndrome de Down e 15 famílias com crianças assintomáticas chegaram à conclusão, por meio de entrevista familiar estruturada, que as famílias as quais possuem crianças autistas e com Síndrome de Down apresentam uma dinâmica familiar diferenciada e, consequentemente, mais estresse se comparadas às famílias com crianças assintomáticas, tendo o score mais significativo nas mães.

Em outro estudo realizado por Gallo-Penna (2006) em Campinas - São Paulo, acerca da QV em mães de pessoas com diagnóstico de TEA, Síndrome de Down, e de pessoas normais, apresentou o resultado $17,12 \%$ para as genitoras de pessoas normais, grupo composto por 23 mães, um resultado considerado bom. Fazendo-se uma comparação entre a presente pesquisa e os estudos citados, percebe-se que, no geral, a QV dos pais de crianças autistas pode ser considerada como boa, não havendo diferenças significativas entre ambos os grupos investigados. 
Tal resultado pode ser compreendido pela capacidade empática e resiliente dos genitores para com os filhos autistas, posto o que já foi mencionado anteriormente acerca do diagnóstico do espectro, segundo o qual traz aos pais sentimentos de perda, bem como tristeza, luto, negação, medo, entre outros, contribuindo para o surgimento de estresse, ansiedade ou depressão que advém comumente de situações novas.

Desta feita, por mais prejuízos que tal diagnóstico venha trazer aos pais, bem como estresse leve e ansiedade moderada, conforme o resultado da presente pesquisa, a família é capaz de reorganizar as mudanças trazidas pelo diagnóstico de TEA e, quando não é possível, a ajuda psicológica é um dos meios que podem contribuir positivamente para a reconstrução emocional desses genitores, posto que o envolvimento familiar nesse processo é um grande passo para o sucesso e QV de toda a família, inclusive dos filhos.

\section{CONCLUSÕES}

O presente estudo preliminar propôs avaliar os níveis de estresse, ansiedade e depressão em pais de crianças diagnosticadas com TEA que realizam acompanhamento no LAPITEA da Universidade CEUMA em São Luís. Ao se estudar os sintomas de cada um e os seus impactos, nota-se que podem comprometer a qualidade de vida dos genitores e afetar o bom funcionamento da dinâmica familiar. No estudo, em questão, observou-se que a intervenção terapêutica realizada no laboratório, possivelmente, tem auxiliado na baixa frequência de tais sintomas nos pais.

Desta feita, é importante que os genitores possam realizar acompanhamento psicológico para que o estado emocional destes venha se manter equilibrado, posto que as crianças em fase de desenvolvimento são dependentes dos cuidados de seus pais quanto ao convívio social, aprendizado, entre outros. Sabe-se que o espectro causa mudanças na família, além de alterar a sua dinâmica. Contudo, é possível conciliar os cuidados do filho autista e ainda possuir uma boa qualidade de vida.

Finalizando, essa pesquisa de campo trouxe resultados positivos mesmo que tenha sido realizada com uma amostra clínica considerada pequena. Logo, faz-se necessário que sejam realizadas outras pesquisas com amostras maiores com o intuito de avaliar com mais profundidade a saúde emocional dos pais de crianças autistas, a fim de proporcionar maior conforto e bem-estar, os quais contribuirão para que haja qualidade de vida no meio familiar. 


\section{REFERÊNCIAS}

WHO - World Health Organization. Depression and other Common Mental Disorders: Global Health Estimates. WHO, 2017.

AMARAL, Kátia C.; CAVALCANTE, Lília I. C.; SILVA, Simone. S. D. C. O Estresse me Mães de Crianças com Autismo: uma revisão de literatura. In: AMARAL, Kátia C. Estresse e percepção de suporte familiar em mães de crianças com autismo, 2013, 91 f. Dissertação (Mestrado em Psicologia) - Universidade Federal do Pará, Belém, 2013, p. 19-44. Disponível em: <http://ppgtpc.propesp.ufpa.br/ARQUIVOS/dissertacoes/K\%C3\%A1tia\%20Carvalho\% 202013.pdf> Acesso em: 23 out. 2017.

AMERICAN PSYCHIATRIC ASSOCIATION. DSM-5: manual diagnóstico e estatístico de transtornos mentais. 5. ed. Porto Alegre: Artmed, 2014. Disponível em: $<$ http://c026204.cdn.sapo.io/1/c026204/cldfile/1426522730/6d77c9965e17b15/b37dfc58aad8cd477904b9bb2ba8a75b/obaudoeduc ador/2015/DSM\%20V.pdf> Acesso em: 23 out. 2017.

BOSA, Cleonice A. Autismo: intervenções psicoeducacionais. Revista Brasileira de Psiquiatria, v. 28, n. 1, p. 47-53, 2006. Disponível em: <http://www.scielo.br/pdf/rbp/v28s1/a07v28s1.pdf> Acesso em: 23 out. 2017.

CASTILLO, Ana Regina G. L. et al. Transtornos de ansiedade. Revista Brasileira de Psiquiatria, São Paulo, v. 22, n. 2, p. 20-23, 2000. Disponível em:

<http://www.scielo.br/pdf/rbp/v22s2/3791.pdf> Acesso em: 18 out. 2017.

CÉSAR, Miria. Autismo afeta cerca de $1 \%$ da população. Disponível em: <http://www.saude.mg.gov.br/ajuda/story/6884-autismo-afeta-cerca-de-1-dapopulacao> Acesso em: 13 out. 2017.

FÁVERO, Maria A. B.; SANTOS, Manoel Antônio D. Autismo infantil e estresse familiar: uma revisão sistemática da literatura. Psicologia: Reflexão e Crítica, Ribeirão Preto, v. 18, n. 3, p. 358-369, 2005. Disponível em:

<http://www.scielo.br/pdf/prc/v18n3/a10v18n3.pdf> Acesso em: 20 out. 2017.

GALLO-PENNA, Eliana C. Qualidade de vida em mães de pessoas com diagnóstico de autismo. Caderno de pós-graduação em distúrbios do desenvolvimento. Universidade Presbiteriana Mackenzie, São Paulo, 2006, p. 1-9. Disponível em: <http://www.mackenzie.br/fileadmin/Pos_Graduacao/Mestrado/Disturbios_do_Desenvo lvimento/Publicacoes/volume_VI/05-2006.pdf> Acesso em 23 out. 2017.

GIL, Antonio C. Como elaborar projetos de pesquisa. 4. ed. São Paulo: Atlas, 2007.

JERUSALINSKY, Alfredo. Psicanálise e desenvolvimento infantil. 4. ed. Porto Alegre: Artes e Ofícios, 2007. 
KINRYS, Gustavo; WYGANT, Lisa E.; Transtornos de ansiedade em mulheres: gênero influência o tratamento? Revista Brasileira de Psiquiatria, São Paulo, v. 27, n. 2, p. 43-50, 2005. Disponível em: 〈http://www.scielo.br/pdf/rbp/v27s2/pt_a03v27s2.pdf> Acesso em: 25 out. 2017.

LEAR, Kathy. Ajude-nos a aprender. Tradução de Margarida Hofmann Windholz et al. 2. ed. Comunidade Virtual Autismo no Brasil, 2004. Disponível em: <http://www.autismo.psicologiaeciencia.com.br/wp-content/uploads/2012/07/Autismoajude-nos-a-aprender.pdf > Acesso em: 23 out. 2017.

SANCHEZ, Abad I. F.; BAPTISTA, Makilin N. Avaliação familiar, sintomatologia depressiva e eventos estressantes em mães de crianças autistas e assintomáticas.

Contextos Clínicos, v. 2, n. 1, p. 40-50, 2009. Disponível em: <http://pepsic.bvsalud.org/pdf/cclin/v2n1/v2n1a05.pdf> Acesso em: 23 out. 2017.

SARDÁ Jr., Jamir J.; LEGAL, Eduardo J.; JABLONSKI Jr, Sérgio J. Estresse: conceitos, métodos, medidas e possibilidades de intervenção. São Paulo: Casa do Psicólogo, 2004.

SCHIMIDT, Carlo; BOSA, Cleonice. Estresse e autoeficácia em mães de pessoas com autismo. Arquivos Brasileiros de Psicolgia, v. 59, n. 2, p. 179-191, 2007. Disponível em: <http://seer.psicologia.ufrj.br/index.php/abp/article/viewFile/88/102> Acesso: 23 out. 2017.

SPROVIERI, Maria Helena S; ASSUMPÇÃO JÚNIOR, Francisco B. Dinâmica familiar de crianças autistas. Arquivo de Neuropsiquiatria, São Paulo, v. 59, n. 2, p. 230-237, 2001. Disponível em:

<http://portal.estacio.br/docs\%5Crevista_estacao_cientifica/02-14.pdf> Acesso em: 22 out. 2017. 